\title{
Existence and Attractiveness of Order One Periodic Solution of a Holling I Predator-Prey Model
}

\author{
Huidong Cheng, Tongqian Zhang, and Fang Wang \\ College of Science, Shandong University of Science and Technology, Qingdao 266510, China \\ Correspondence should be addressed to Huidong Cheng, chd900517@sdust.edu.cn
}

Received 16 April 2012; Accepted 13 August 2012

Academic Editor: Narcisa C. Apreutesei

Copyright (c) 2012 Huidong Cheng et al. This is an open access article distributed under the Creative Commons Attribution License, which permits unrestricted use, distribution, and reproduction in any medium, provided the original work is properly cited.

According to the integrated pest management strategies, a Holling type I functional response predator-prey system concerning state-dependent impulsive control is investigated. By using differential equation geometry theory and the method of successor functions, we prove the existence of order one periodic solution, and the attractivity of the order one periodic solution by sequence convergence rules and qualitative analysis. Numerical simulations are carried out to illustrate the feasibility of our main results which show that our method used in this paper is more efficient than the existing ones for proving the existence and attractiveness of order one periodic solution.

\section{Introduction}

It is one of the significant problems in the world today to prevent plant pests and pesticide pollution and to protect ecological balance for the sustainable development of agriculture and forestry, which is also an important research topic demanding prompt solution. In recent decades, plant pests worldwide are increasingly serious with the damage of the world's natural ecosystems. In agricultural production, pesticides spaying (chemical control) and release of natural enemies (biological control) are the ways commonly used for pest control. But if we implement chemical control as soon as pests appear, many problems are caused: the first is environmental pollution; the second is increase of costs including human and material resources and time; the third is killing natural enemies, such as parasitic wasp; the last is pests' resistance to pesticides, which brings great negative effects [1-3]. The second way, which controls pests with the help of the increasing natural enemies, can avoid problems caused by chemical control and gets more and more attention. So many scholars have been studying and discussing it [4-6]. 
Considering the effectiveness of the chemical control and nonpollution and limitations of the biological one, people have proposed the method of integrated pest management (IPM), which is a pest management system integrating all appropriate ways and technologies to control economic injury level (EIL) caused by pest populations in view of population dynamics and its relevant environment. In the process of practical application, people usually implement the following two schemes for the integrated pest management: one is to implement control at a fixed time to eradicate pests $[7,8]$; the other is to implement measures only when the amount of pests reaches a critical level, which is to make the amount less than certain economic impairment level, not to wipe out pests [9-11].

In consideration of predator-prey capacity, Holling [12] proposes three different predations with functional response based on experiments; the average predator-prey system with Holling response is as follows:

$$
\begin{aligned}
& x^{\prime}(t)=x g(x)-y \phi(x), \\
& y^{\prime}(t)=-d y+e y \phi(x),
\end{aligned}
$$

where $x$ represents the densities for prey's density while $y$ is the predator's; $g(x)$ is the unit rate of prey density in lack of predators. $\phi(x)$ is the Holling functional response. Holling type-I functional response is

$$
\phi(x)= \begin{cases}c x, & x \leq x_{0} \\ c x_{0}, & x>x_{0}\end{cases}
$$

where $C$ is a constant; when the amount of prey is greater than certain threshold value $x_{0}$, predatory rate is a constant.

Holling type-II and type-III functional responses are as follows, respectively:

$$
\phi(x)=\frac{\alpha x}{1+\omega x}, \quad \phi(x)=\frac{\alpha x^{2}}{\beta+x^{2}} .
$$

Refer to [12] for details.

As the Lotka-Volterra predator-prey system with Holling functional response is more practical, many authors have studied about it [13-15]. The researches mostly focus on LotkaVolterra predator-prey model with Holling type-II or Holling type-III functional response in contrast to the model with Holling type-I. This paper sets up and adopts new mathematic method to study comprehensive control (releasing natural enemies and spraying pesticide) 
adopted when the amount of predators (pests) reaches the economic test (ET), thus the following pest-control model with Holling type-I functional response is set up:

$$
\begin{gathered}
x^{\prime}(t)=r x(t)-c x(t) y(t), \quad x \leq x_{0}, \quad x<h, \\
y^{\prime}(t)=-d y(t)+e c x(t) y(t), \quad x \leq x_{0}, \quad x<h, \\
x^{\prime}(t)=r x(t)-c x_{0} y(t), \quad x>x_{0}, \quad x<h, \\
y^{\prime}(t)=-d y(t)+e c x_{0} y(t), \quad x>x_{0}, \quad x<h, \\
\Delta x(t)=-\alpha x(t), \quad x=h, \\
\Delta y(t)=-\beta y(t)+q, \quad x=h,
\end{gathered}
$$

where $r, c, d, e$ are all positive constants, $x(t)$ and $y(t)$ represent the densities of prey (pest) and predator (natural enemy) at time $t$, respectively; $r$ is the intrinsic growth rate of the prey; $d$ denotes the death rate of the predator; $h$ is the economic threshold ET; $\alpha, \beta \in(0,1)$ represent the proportion of killed prey and predator by spraying pesticides, respectively, $q>$ 0 is the number of natural enemies released at this time. When the amount of prey reaches the threshold $h$ at time $t_{h}$, controlling measures are taken and the amount of prey and predator abruptly turns to $(1-\alpha) h$ and $(1-\beta) y\left(t_{h}\right)+q$, respectively. $\Delta x=x\left(t^{+}\right)-x(t), \Delta y=y\left(t^{+}\right)-$ $y(t), x\left(t^{+}\right)=\lim _{w \rightarrow 0^{+}} x(t+w), y\left(t^{+}\right)=\lim _{w \rightarrow 0^{+}} y(t+w)$.

This paper is organized as follows. In the next section, we present some basic definitions and important lemmas as preliminaries. In Section 3, we prove existence for an order one periodic solution of system (1.4). The sufficient conditions for the attractiveness of order one periodic solutions of system (1.4) are obtained in Section 4. At last, we state conclusion and the main results are carried out to illustrate the feasibility by numerical simulations.

\section{Preliminaries}

We first consider the model (1.4) without impulse effects

$$
\begin{gathered}
x^{\prime}(t)=r x(t)-c x(t) y(t), \quad x \leq x_{0}, \\
y^{\prime}(t)=-d y(t)+e c x(t) y(t), \quad x \leq x_{0}, \\
x^{\prime}(t)=r x(t)-c x_{0} y(t), \quad x>x_{0}, \\
y^{\prime}(t)=-d y(t)+e c x_{0} y(t), \quad x>x_{0} .
\end{gathered}
$$

We consider the following function:

$$
V(x, y)=\int_{x^{*}}^{x} \frac{-d+e \phi(s)}{\phi(s)} d s+\int_{y^{*}}^{y} \frac{s-y^{*}}{s} d s,
$$

and we can easily know that $V(x, y)$ is positive definite in the first quartile and fits for all conditions of Liapunov function. 
We can get that

$$
V^{\prime}(x, y)=\frac{e x y^{*}}{\phi(x)}\left(\phi(x)-\phi\left(x^{*}\right)\right)\left(\frac{\phi\left(x^{*}\right)}{x^{*}}-\frac{\phi(x)}{x}\right) .
$$

It is easily proved that $V^{\prime}(x, y) \equiv 0$ on condition that $x \leq x_{0}$, so all solutions of model (1.4) which form a set $\left\{(x, y) / V(x, y) \leq V\left(x_{0}, y^{*}\right)\right\}$ are closed trajectory $V(x, y)=C$, where $0<C<V\left(x_{0}, y^{*}\right)$.

Since $V I(x, y)>0$ on condition that $x>x_{0}$, so the trajectory of system (2.1) passes through closed curve $V(x, y)=C$ when it is out of the curve $V(x, y)=V\left(x_{0}, y^{*}\right)$.

Therefore, we observe the straight line:

$$
L(x, y)=y+x-n, \quad n>0, \quad x_{0}<x \leq h .
$$

The derivative of $L(x, y)$ along (2.1) is that

$$
\begin{aligned}
L^{\prime}(x, y) / L=0 & =x \prime+y^{\prime}=-d y+e c x_{0} y+r x-e c x_{0} \\
& =-\left(d n-e c x_{0} n+c x_{0} n+c x_{0} h-d x_{0}\right)-\left(e c x_{0}-r-c x_{0}\right) \\
& \leq d h-e c x_{0}^{2}+r h+c x_{0} h-\left(d-e c x_{0}+c x_{0}\right) n
\end{aligned}
$$

We have $L /{ }_{L=0}<0$ on condition that $n>\left(d h-e c x_{0}^{2}+r h+c x_{0} h\right) /\left(d-e c x_{0}+c x_{0}\right)$. Therefore, we can get the following lemma.

Lemma 2.1. The system (2.1) possesses the following.

(I) Two steady states $0(0,0)$-saddle point, and $R(d / e c, r / c)=R\left(x^{*}, y^{*}\right)$-stable centre under the condition $x \leq x_{0}$ and $d \leq e c x_{0}$.

(II) The trajectory of system (2.1) goes across the straight line $y+x-n=0$ from the right to the left under condition $x_{0} \leq x \leq h$ and $n>\left(d h-e c x_{0}^{2}+r h+c x_{0} h\right) /\left(d-e c x_{0}+c x_{0}\right)$, and intersects with the straight line $x=x_{0}$.

Definition 2.2. A triple $\left(X, \Pi, R^{+}\right)$is said to be a semi-dynamical system if $X$ is a metric space $R^{+}$is the set of all non-negative real and $\Pi(P, t): X \times R^{+} \rightarrow X$ is a continuous map such that;

(i) $\Pi(P, 0)=P$ for all $P \in X$;

(ii) $\Pi(P, t)$ is continuous for $t$ and $s$;

(iii) $\Pi(\Pi(P, t))=\Pi(P, t+s)$ for all $P \in X$ and $t, s \in R^{+}$. Sometimes a semi-dynamical system $\left(X, \Pi, R^{+}\right)$is denoted by $(X, \Pi)$.

Definition 2.3. Assume that

(i) $(X, \Pi)$ is a semi-dynamical system;

(ii) $M$ is a nonempty subset of $X$;

(iii) function $I: M \rightarrow X$ is continuous, and, for any $P \in M$, there exists a $\varepsilon>0$ such that for any $0<|t|<\varepsilon, \Pi(P, t) \notin M$. 


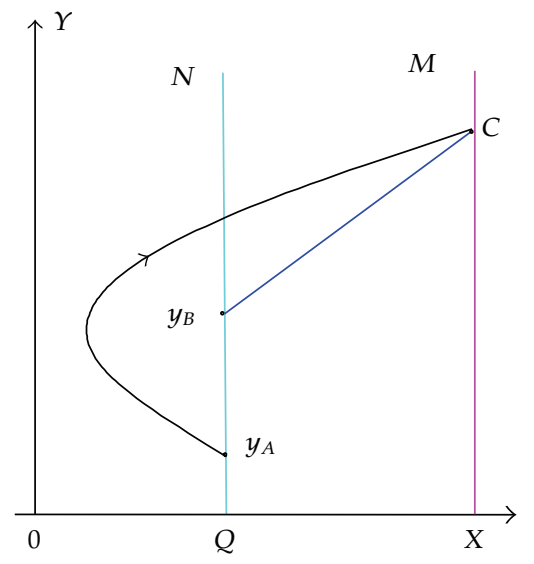

Figure 1

Then, $(X, \Pi, M, I)$ is called an impulsive semi-dynamical system.

For any $P$ the function $\Pi_{P}: R^{+} \rightarrow X$ defined as $\Pi_{P}(t)=\Pi(P, t)$ is continuous and we call $\Pi_{P}(t)$ the trajectory passing through point $P$. The set $C^{+}(P)=\{\Pi(P, t) / 0 \leq t<+\infty\}$ is called positive semitrajectory of point $P$. The set $C^{-}(P)=\{\Pi(P, t) /-\infty<t \leq 0\}$ is called the negative semi-trajectory of point $P$.

Definition 2.4. We consider state-dependent impulsive differential equations:

$$
\begin{array}{ll}
x^{\prime}(t)=P(x, y), & (x, y) \notin M(x, y), \\
y^{\prime}(t)=Q(x, y), & (x, y) \notin M(x, y), \\
\Delta x(t)=\alpha(x, y), & (x, y) \in M(x, y), \\
\Delta y(t)=\beta(x, y), & (x, y) \in M(x, y),
\end{array}
$$

where $M(x, y)$ and $N(x, y)$ represent the straight line or curve line on the plane and $M(x, y)$ is called impulsive set. The function $I$ is continuous mapping, and $I(M)=N, I$ is called the impulse function. $N(x, y)$ is called the phase set. We define "dynamic system" constituted by the definition of solution of state impulsive differential equation (2.5) as "semi-continuous dynamic system," which is denoted as $(\Omega, f, I, M)$.

Definition 2.5. Suppose that the impulse set $M$ and the phase set $N$ are both lines, as shown in Figure 1. Define the coordinate in the phase set $N$ as follows: denote the point of intersection $Q$ between $N$ and $x$-axis as $O$, then the coordinate of any point in $N$ is defined as the distance between $A$ and $Q$ and is denoted by $y_{A}$. Let $C$ denote the point of intersection between the trajectory starting from $A$ and the impulse set $M$, and let $B$ denote the phase point of $C$ after impulse with coordinate $y_{B}$. Then we define $B$ as the successor point of $A$, and then the successor function of point $A$ is that $f(A)=y_{B}-y_{A}$.

Definition 2.6. A trajectory $\widetilde{\Pi}\left(P_{0}, t\right)$ is called order one periodic solution with period $T$ if there exists a point $P_{0} \in N$ and $T>0$ such that $P=\Pi\left(P_{0}, t\right) \in M$ and $P^{+}=I(P)=P_{0}$. 
We get these lemmas from the continuity of composite function and the property of continuous function.

Lemma 2.7. Successor function defined in Definition 2.2 is continuous.

Lemma 2.8. In system (1.4), if there exist $A \in N, B \in N$ satisfying successor function $f(A) f(B)<$ 0 , then there must exist a point $P(P \in N)$ satisfying $f(P)=0$ : the function between the point of $A$ and the point of $B$, thus there is an order one periodic solution in system (1.4).

\section{Existence of the Order One Periodic Solution}

In this section we shall investigate the existence of an order one periodic solution of system (1.4) by using the successor function defined in this paper. For this goal, we denote that

$$
\begin{aligned}
& M_{1}=\left\{(x, y) \in R_{2}^{+} \mid x=h, h<x_{0}, y \geq 0\right\} \\
& M_{2}=\left\{(x, y) \in R_{2}^{+} \mid x=h, h>x_{0}, y \geq 0\right\}
\end{aligned}
$$

Phase set $N$ of set $M$ is that

$$
N=I(M)=\left\{(x, y) \in R_{2}^{+} \mid x=(1-\alpha) h, y \leq q\right\}
$$

Isoclinic line is denoted, respectively, by lines

$$
\begin{aligned}
& L_{1}=\left\{(x, y)(x, y) \in R_{2}^{+} \mid y=\frac{r}{c}, 0 \leq x \leq x_{0}\right\}, \\
& L_{2}=\left\{(x, y) \in R_{2}^{+} \mid x=\frac{d}{e c}, 0 \leq x \leq x_{0}, y \geq 0\right\}, \\
& L_{3}=\left\{(x, y) \in R_{2}^{+} \mid y=\frac{r}{c x_{0}} x, x \geq x_{0}, y \geq \frac{r}{c}\right\} .
\end{aligned}
$$

For the convenience, if $P \in \Omega-M, F(P)$ is defined as the first point of intersection of $C^{+}(P)$ and $M$, that is, there exists a $t_{1} \in R_{+}$such that $F(P)=\Pi\left(P, t_{1}\right) \in M$, and for $0<t<t_{1}, \Pi(P, t) \notin$ $M$; if $B \in N, R(B)$ is defined as the first point of intersection of $C^{-}(P)$ and $N$, that is there exists a $t_{2} \in R_{+}$such that $R(B)=\Pi\left(B,-t_{2}\right) \in N$, and for $-t<t<0, \Pi(B, t) \notin N$.

For any point $P$, we denote $y_{P}$ as its ordinate. If the point $P\left(h, y_{P}\right) \in M$, pulse shall occur at the point $P$, and the impulsive function transfers the point $P$ into $P^{+} \in N$. Without loss of generality, we assume that the initial point of the trajectory lies in phase set $N$ unless otherwise specified.

Due to the practical significance, in this paper we assume the set $N$ always lies in the left side of stable centre $R$, that is, $(1-\alpha) h<d / e c$.

In the light of the different position of the set $M_{1}$ and the set $M_{2}$, we consider the following three cases.

Case $1(0<h<d / e c)$. In this case, sets $M_{1}$ and $N$ are both in the left side of stable center $R$. The line $L_{1}$ intersects with $N$ at point $A((1-\alpha) h, r / c)$, as shown in Figure 2 . The trajectory 


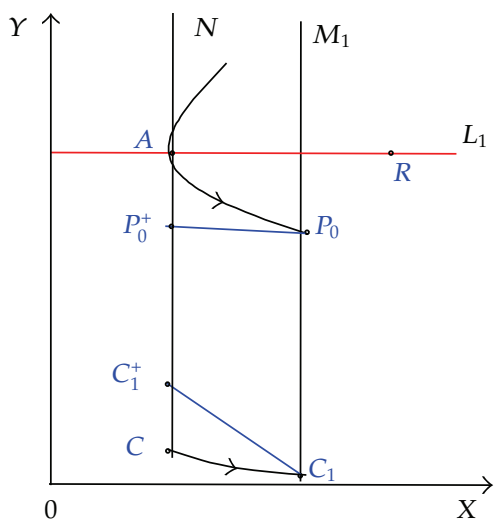

Figure 2

passing through point $A$ which tangents to the set $N$ at point $A$ intersects with the set $M_{1}$ at point $P_{0}\left(h, y_{P_{0}}\right)$. Since the point $P_{0} \in M_{1}$, impulse occurs at point $P_{0}$, supposing point $P_{0}$ is subject to impulsive effects to point $P_{0}^{+}\left((1-\alpha) h, y_{P_{0}^{+}}\right)$, where $y_{P_{0}^{+}}=(1-\beta) y_{P_{0}}+q$, and the position of the point $P_{0}^{+}$has the following three cases.

Subcase $1.1\left((1-\beta) y_{P_{0}}+q<r / c\right)$. In this case, the point $P_{0}^{+}$lies below the point $A$, thus the successor function of the point $A$ is $f(A)=(1-\beta) y_{P_{0}}+q-r / c<0$.

Take another point $C((1-\alpha) h, \varepsilon) \in N$, where $\varepsilon>0$ is small enough. Then there must exist a trajectory passing through the point $C$ which intersects $M_{1}$ at point $C_{1}\left(h, y_{C_{1}}\right) \in M_{1}$. Supposing that the point $C_{1}$ is subject to impulsive effects to point $C_{1}^{+}\left((1-\alpha) h, y_{C_{1}^{+}}\right), y_{C_{1}^{+}}=$ $(1-\beta) y_{C_{1}}+q>\varepsilon$, so we have $f(C)=y_{C_{1}^{+}}-\varepsilon>0$.

By Lemma 2.8, there exists an order one periodic solution of system (1.4), whose initial point is between the point $C$ and the point $A$ in set $N$.

Subcase $1.2\left((1-\beta) y_{P_{0}}+q>r / c\right)$ (as shown in Figure 3$)$. In this case, the point $P_{0}^{+}$lies above the point $A$; there must exist a trajectory passing through the point $P_{0}^{+}$which intersects the set $M_{1}$ at a point $P_{2}\left(h, y_{P_{2}}\right)$ under $P_{1}$. Denote the coordinates of impulsive point $P_{2}^{+}\left((1-\alpha) h, y_{P_{2}^{+}}\right)$ corresponding to the point $P_{2}\left(h, y_{P_{2}}\right)$, then $y_{P_{2}^{+}}<y_{P_{0}^{+}}$. So we obtain $f\left(P_{0}^{+}\right)=y_{P_{2}^{+}}-y_{P_{0}^{+}}<0$.

Take that another point $B_{1}((1-\alpha) h, \varepsilon+r / c) \in N$ above $A$, where $\varepsilon>0$ is small enough. Then there must exist a trajectory passing through the point $B_{1}$ which intersects $M_{1}$ at a point $P_{1}\left(h, y_{P_{1}}\right)$. In view of continuous dependence of the solution on initial value and time, we know $y_{P_{1}}<y_{P_{0}}$ and the point $P_{1}$ is close to $P_{0}^{+}$enough, then we obtain $f\left(B_{1}\right)=y_{P_{1}^{+}}-y_{B_{1}}>0$.

From Lemma 2.8, there exists an order one periodic solution of system (1.4), whose initial point is between $B_{1}$ and $P_{0}^{+}$in set $N$.

Subcase $1.3\left((1-\beta) y_{P_{0}}+q=r / c\right) . P_{0}^{+}$coincides with $A$, and the successor function of $A$ is $f(A)=0$, so there exists an order one periodic solution of system (1.4) which is just a part of the trajectory passing through the point $A$.

Now we can summarize the above results as the following theorem.

Theorem 3.1. Assuming that $d<e c x_{0}, 0<h \leq d / e c<x_{0}$, there exists an order one periodic solutions of the system (1.4).

Case $2\left(d / e c<h \leq x_{0}\right)$. In this case, the set $M_{1}$ is in the right side of $R$ and set $N$ is in the left side of $R$. Let the line $L_{1}$ intersects the sets $M_{1}$ and $N$ at points $A(h, r / c)$ and $B((1-\alpha) h, r / c)$, 


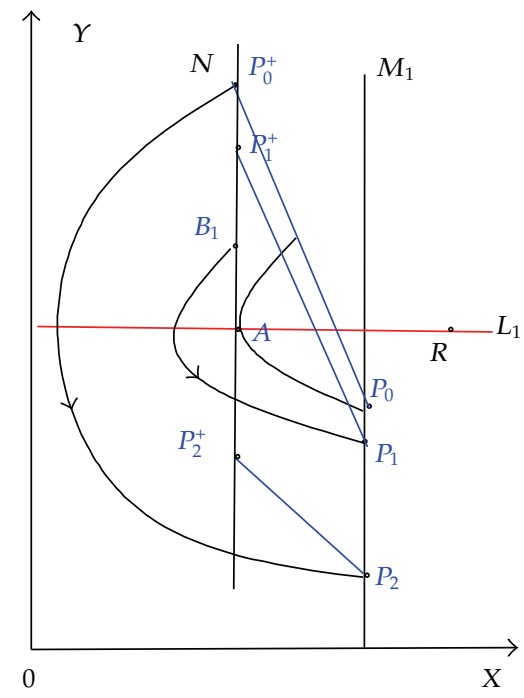

Figure 3

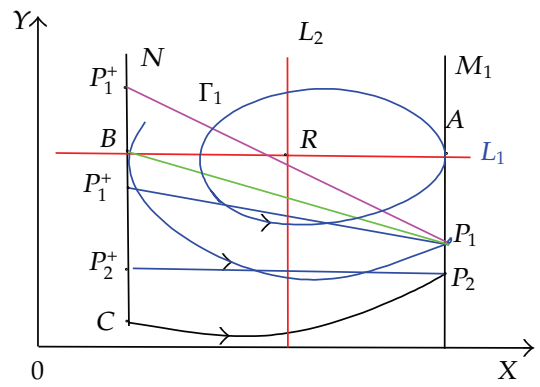

Figure 4

respectively (Figure 4), then there exists a unique closed trajectory $\Gamma_{1}$ of system (1.4) which is contained inside the point $R$ and is tangent to the set $M_{1}$ at the point $A$.

Since $\Gamma_{1}$ is a closed trajectory, we take their minimal value of abscissas $\delta_{\min }$ at the trajectory $\Gamma_{1}$, namely, $\delta_{\min } \leq x$ holds for any abscissas of $\Gamma_{1}$.

In light of the different positions of the set $N$, we consider the following three subject cases.

Subcase $2.1\left(0<(1-\alpha) h<\delta_{\min }<d / e c\right)$. In this case, there is a trajectory, which is contained inside the point $R$ and is tangent to the set $N$ at point $B$ which intersects $M_{1}$ at the point $P_{1}\left((1-\alpha) h, y_{P_{1}}\right) \in M_{1}$. Supposing that point $P_{1}$ is subject to impulsive effects to point $P_{1}^{+}((1-$ $\left.\alpha) h, y_{P_{1}^{+}}\right) \in N$, here $y_{P_{1}^{+}}=(1-\beta) y_{P_{1}}+q$. Like the analysis of Case 1 we can prove there exists an order one periodic solution in system (1.4) in this case.

Now we can summarize the above results as the following theorem.

Theorem 3.2. If $d<e c x_{0}, 0<(1-\alpha) h<\delta_{\min }<d / e c<h \leq x_{0}$, there exists an order one periodic solution in system (1.4). 
Subcase $2.2\left(\delta_{\min }<(1-\alpha) h<d / e c\right)$. In this case, let the closed trajectory $\Gamma_{1}$ of system (1.4) intersects the set $N$ at two points $A_{1}\left((1-\alpha) h, y_{A_{1}}\right)$ and $A_{2}\left((1-\alpha) h, y_{A_{2}}\right)$, as shown in Figure 5 . Since $A \in M_{1}$, impulse occurs at point $A$. Supposing that point $A$ is subject to impulsive effects to point $P_{0}^{+}\left((1-\alpha) h, y_{P_{0}^{+}}\right)$, here $y_{P_{0}^{+}}=(1-\beta)(r / c)+q$.

If $(1-\beta)(r / c)+q=y_{A_{1}}$ or $(1-\beta)(r / c)+q=y_{A_{2}}, P_{0}^{+}$coincides with $A_{1}$ or $P_{0}^{+}$coincides with $A_{2}$; the successor function of $A_{1}$ or $A_{2}$ is that $f\left(A_{1}\right)=0$ or that $f\left(A_{2}\right)=0$, so there exists an order one periodic solution of system (1.4) which is just a part of the trajectory $\Gamma_{1}$.

If $(1-\beta)(r / c)+q<y_{A_{2}}$, the point $P_{0}^{+}$lies below the point $A_{2}$, like the analysis of Subcase 1.1 , and we can prove there exists an order one periodic solution to the system (1.4) in this case.

If $(1-\beta)(r / c)+q>y_{A_{1}}$, the point $P_{0}^{+}$is above the point $A_{1}$. Like the analysis of Subcase 1.2 , we can prove there exists an order one periodic solution to the system (1.4) in this case.

Now we can summarize the above results as the following theorem.

Theorem 3.3. Assuming that $d \leq e c x_{0}, \delta_{\min }<(1-\alpha) h<d / e c<h<x_{0}$, if $(1-\beta)(r / c)+q \geq y_{A_{2}}$ or $(1-\beta)(r / c)+q \geq y_{A_{1}}$, then there exists an order one periodic solution to the system (1.4).

Subcase $2.3\left(y_{A_{2}}<(1-\beta)(r / c)+q<y_{A_{1}}\right)$. In this case, we note that the point $P_{0}^{+}$must lie between the point $A_{1}$ and the point $A_{2}$ (as shown in Figure 6). Taking a point $E_{1} \in M_{1}$ such that $E_{1}$ jumps to $A_{2}$ after the impulsive effect, we denote $A_{2}=E_{1}^{+}$. Since $y_{P_{0}^{+}}>y_{E_{1}^{+}}$, we have $y_{A}>y_{E_{1}}$. Let $R\left(E_{1}\right)=E_{2}^{+} \in N$, taking a point $E_{2} \in M_{1}$ such that $E_{2}$ jumps to $E_{2}^{+}$after the impulsive effects, then we have $y_{E_{1}^{+}}>y_{E_{2}^{+}}, y_{E_{1}}>y_{E_{2}}$. This process continues until there exists a $E_{K}^{+} \in N\left(K \in Z_{+}\right)$satisfying $y_{E_{K}^{+}}<q$. So we obtain a sequence $\left\{E_{k}\right\}_{k=1,2, \ldots, K}$ of set $M_{1}$ and a sequence $\left\{E_{k}^{+}\right\}_{k=1,2, \ldots, K}$ of set $N$ satisfying $R\left(E_{k-1}\right)=E_{k}^{+} \in N, y_{E_{k-1}^{+}}>y_{E_{k}^{+}}$. In the following, we will prove the trajectory of system (1.4) with any initiating point of set $N$ which will ultimately stay in $\Gamma_{1}$.

From the vector field of system (1.4), we know the trajectory of system (1.4) with any initiating point between the points $A_{1}$ and $A_{2}$ will be free from impulsive effect and ultimately will stay in $\Gamma_{1}$.

For any point below $A_{2}$, it must lie between $E_{k}^{+}$and $E_{k-1}^{+}$, where $k=2,3, \ldots, K+1$ and $A_{2}=E_{1}^{+}$. After $k$ times' impulsive effects, the trajectory with this initiating point will arrive at some point of the set $N$ which must be between $A_{1}$ and $A_{2}$, and then ultimately stay in $\Gamma_{1}$.

The trajectory with any initiating point above $A_{1}$ will intersect set $N$ at some point below $A_{2}$ with time increasing, so just like the analysis above we obtain that it will ultimately stay in $\Gamma_{1}$.

Now we can summarize the above results as the following theorem.

Theorem 3.4. Assuming that $d \leq e c x_{0}$ and $\delta_{\min }<(1-\alpha) h<d / e c<h \leq x_{0}$, if $y_{A_{2}}<(1-$ $\beta)(r / c)+q<y_{A_{1}}$, there is no periodic solution in system (1.4) and the trajectory with any initiating point of set $N$ will stay in $\Gamma_{1}$.

Case $3\left(0<(1-\alpha) h<d / e c<x_{0}<h\right)$. In this case, denote the intersection of the line $L_{1}$ and the set $N$ by point $B((1-\alpha) h, r / c)$, and the intersection of the line $L_{3}$ and the set $M_{2}$ by point $A\left(h, r h / c x_{0}\right)$ (as shown in Figure 7). Through Lemma 2.8 and qualitative analysis, there exists a unique closed trajectory $\Gamma_{2}$ of system (1.4) which is tangent to the set $M_{2}$ at the point $A$ and has minimal value $\lambda_{\min }$ at the line $L_{1}$. In the light of the different position of the set $N$, we consider the following two cases. 


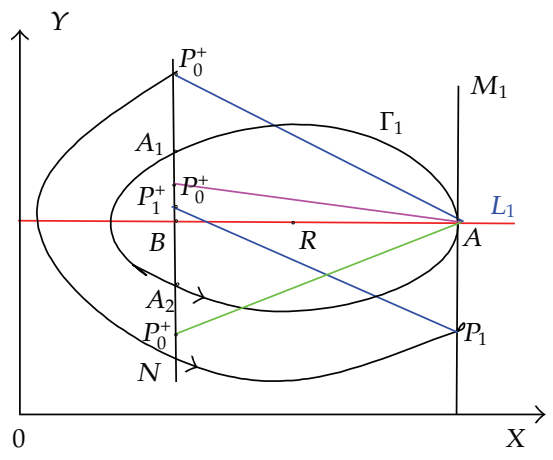

Figure 5

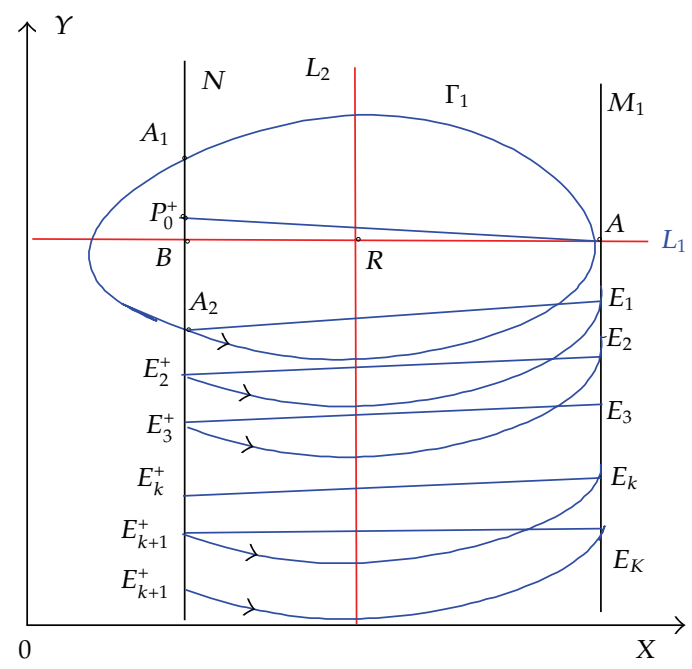

Figure 6

Subcase $3.1\left(0<(1-\alpha) h<\lambda_{\min }\right)$. In this case, there exists a unique trajectory of system (1.4) which is tangent to the set $N$ at the point $B$. Set $F(B)=P_{1} \in M_{2}$, then pulse occurs at point $P_{1}$, and the impulsive function transfers the point $P_{1}$ into $P_{1}^{+}$. Like the analysis of Subcase 2.1, we can prove there exists an order one periodic solution in system (1.4) in this case.

Subcase $3.2\left(\lambda_{\min }<(1-\alpha) h<x_{0}<h\right)$. In this case, let the closed trajectory $\Gamma_{2}$ of system (1.4) intersect with $N$ at two points $A_{1}\left((1-\alpha) h, y_{A_{1}}\right)$ and $A_{2}\left((1-\alpha) h, y_{A_{2}}\right)$. Like the analysis of Subcase 2.2 we can prove there exists an order one periodic solution in system (1.4) in this case; like the analysis of Subcase 2.3, we can prove there is no periodic solution in system (1.4) and the trajectory with any initiating point of set $N$ will stay in $\Gamma_{2}$.

\section{Attractiveness of the Order One Periodic Solutions}

In this section, under the condition of existence of order one periodic solution to system (1.4) and the initial value of pest population $x(0) \leq h$, we discuss its attractiveness. We focus on Case 1, and by similar method we can obtain similar results about Cases 2 and 3 . 


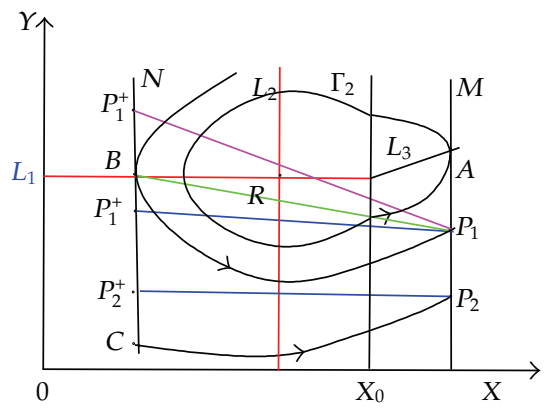

Figure 7

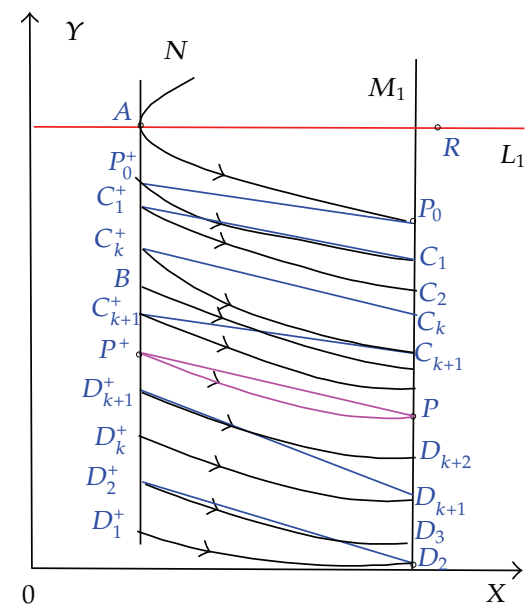

Figure 8

Theorem 4.1. If system (1.4) satisfies the condition of Theorem 3.1 and $y_{P_{0}^{+}}<y_{A}$, then

(I) there exists an odd number of order one periodic solutions of system (1.4) with initial value between $C$ and $A$ in the set $N$

(II) if the periodic solution is unique, then the periodic solution is attractive in region $\Omega_{1}=$ $\{(x, y) \mid x \geq 0, y \geq 0, x(0) \leq h\}$.

Proof. (I) According to the Subcase 1.3, $f(A)<0$ and $f(c)>0$ (see Figure 2); the successor function $f(x)$ is continuous, so there exists an odd number of root satisfying $f(x)=0$, then we can get that there exists an odd number of order one periodic solutions of system (1.4) with initial value between $C$ and $A$ in the set $N$.

(II) By the derivation of Theorem 3.1, we know there exists an order one periodic solution of system (1.4). We assume trajectory $\widehat{P^{+} P}$ and segment $\overline{P P^{+}}$formulate a unique order one periodic solution of system (1.4) with initial point $P^{+} \in N$ (Figure 8).

On the one hand, take a point $D_{1}^{+}((1-\alpha) h, \varepsilon) \in N$ satisfying $y_{D_{1}^{+}}=\varepsilon<q$ and $y_{D_{1}^{+}}<$ $y_{P^{+}}$. The trajectory passes through the point $D_{1}^{+}((1-\alpha) h, \varepsilon)$ which intersects set $M_{1}$ at point $D_{2}\left(h, y_{D_{2}}\right)$, that is, $F\left(D_{1}^{+}\right)=D_{2} \in M_{1}$, then we have $y_{D_{2}}<y_{P}$, thus $y_{D_{2}^{+}}<y_{P^{+}}$. Since $y_{D_{2}^{+}}=$ $(1-\beta) y_{D_{2}}+q>\varepsilon$, so we obtain $f\left(D_{1}^{+}\right)=y_{D_{2}^{+}}-y_{D_{1}^{+}}=y_{D_{2}^{+}}-\varepsilon>0$; set $F\left(D_{2}^{+}\right)=D_{3} \in M_{1}$, because $y_{D_{1}^{+}}<y_{D_{2}^{+}}<y_{P^{+}}$; we know $y_{D_{2}}<y_{D_{3}}<y_{P}$, then we have $y_{D_{2}^{+}}<y_{D_{3}^{+}}<y_{P^{+}}$and 
$f\left(D_{2}^{+}\right)=y_{D_{3}^{+}}-y_{D_{2}^{+}}>0$. This process is continuous, then we get a sequence $\left\{D_{k}^{+}\right\}_{k=1,2 \ldots}$ of set $N$ satisfying

$$
y_{D_{1}^{+}}<y_{D_{2}^{+}}<\cdots<y_{D_{k}^{+}}<\cdots<y_{P^{+}}
$$

and $f\left(D_{k}^{+}\right)=y_{D_{k+1}^{+}}-y_{D_{k}^{+}}>0$. Series $\left\{y_{D_{k}^{+}}\right\}_{k=1,2, \ldots}$ increase monotonously and have upper bound, so that $\lim _{k \rightarrow \infty} y_{D_{k}^{+}}$exists.

Next we will prove $\lim _{k \rightarrow \infty} y_{D_{k}^{+}}=y_{P^{+}}$. Setting $\lim _{k \rightarrow \infty} D_{k}=D^{+}$, we will prove $P^{+}=D^{+}$. Otherwise $P^{+} \neq D^{+}$, then there is a trajectory passing through the point $D^{+}$which intersects the set $M_{1}$ at point $\tilde{D}$, then we have $y_{\tilde{D}}<y_{P}, y_{\tilde{D}^{+}}<y_{P^{+}}$. Since $f\left(D^{+}\right) \geq 0$ and $P^{+} \neq D^{+}$, according to the uniqueness of the periodic solution, then we have $f\left(D^{+}\right)=y_{\tilde{D}^{+}}-y_{D^{+}}>0$, thus $y_{D^{+}}<y_{\tilde{D}^{+}}<y_{P^{+}}$hold. Set $F\left(\tilde{D}^{+}\right)=\widetilde{\widetilde{D}} \in M_{1}$. In view of vector field and disjointness of any two trajectories, we know $y_{\tilde{D}}<y_{\tilde{D}}<y_{P}$ and $y_{\tilde{D}^{+}}<y_{\tilde{D}^{+}}<y_{P^{+}}$, then we have $f\left(\widetilde{D}^{+}\right)=y_{\tilde{D}} \tilde{\tilde{D}}^{+} y_{\tilde{D}^{+}}>0$; this contradicts the fact that $D^{+}$is a limit of sequence $\left\{D_{k}^{+}\right\}_{k=1,2, \ldots}$, so we obtain $P^{+}=D^{+}$and $\lim _{\mathrm{k} \rightarrow \infty} y_{D_{k}^{+}}=y_{P^{+}}$.

On the other hand, set $F\left(P_{0}^{+}\right)=C_{1} \in M_{1}$, then $C_{1}$ jumps to $C_{1}^{+} \in N$ under the impulsive effects. Since $y_{P^{+}}<y_{P_{0}^{+}}<y_{A}$, we have $y_{P}<y_{C_{1}}<y_{P_{0}}$, thus we obtain that $y_{P^{+}}<y_{C_{1}^{+}}<y_{P_{0}^{+}}$ and that $f\left(P_{0}^{+}\right)=y_{C_{1}^{+}}-y_{P_{0}^{+}}<0$. Set $F\left(C_{1}^{+}\right)=C_{2} \in M_{1}$, then $C_{2}$ jumps to $C_{2}^{+} \in N$ under the impulsive effects. We have $y_{P^{+}}<y_{C_{2}^{+}}<y_{C_{1}^{+}}, f\left(C_{1}^{+}\right)=y_{C_{2}^{+}}-y_{C_{1}^{+}}<0$; this process is continuous, and we can obtain a sequence $\left\{C_{k}^{+}\right\}_{k=1,2, \ldots}$ of set $N$ satisfying

$$
y_{P_{0}^{+}}>y_{C_{1}^{+}}>y_{C_{2}^{+}}>\cdots>y_{C_{k}^{+}}>\cdots>y_{P^{+}}
$$

and $f\left(C_{k}^{+}\right)=y_{C_{k+1}^{+}}-y_{C_{k}^{+}}<0$. Series $\left\{y_{C_{k}^{+}}\right\}_{k=1,2, \ldots}$ decrease monotonously and have lower bound, so that $\lim _{k \rightarrow \infty} y_{C_{k}^{+}}$exists. Similarly, we can prove that $\lim _{k \rightarrow \infty} y_{C_{k}^{+}}=y_{P}^{+}$.

Since the trajectory initiating any point of $\Omega_{1}=\{(x, y) \mid x \geq 0, y \geq 0, x(0) \leq h\}$ will certainly intersect with set $N$, next we only need to prove the trajectory initiating any point of $N$ will ultimately tend to be the unique order one periodic solution.

Any point $B \in N$ below $A$ must be in some interval $\left[y_{C_{k+1}^{+}}, y_{C_{k}^{+}}\right)_{k=1,2, \ldots},\left[y_{C_{1}^{+}}, y_{P_{0}^{+}}\right),\left[y_{P_{0}^{+}}, y_{A}\right),\left[y_{D_{k}^{+}}, y_{D_{k+1}^{+}}\right)_{k=1,2, \ldots}$. Without loss of generality, we assume the point $B \in\left[y_{C_{k+1}^{+}}, y_{C_{k}^{+}}\right)$. The trajectory with initiating point $B$ moves between trajectories $\widehat{C_{k}^{+} C_{k+1}}$ and $\widehat{C_{k+1}^{+} C_{k+2}}$ and intersects with $M_{1}$ at some point between $C_{k+2}$ and $C_{k+1}$, and under the impulsive effects it jumps to the point of $N$ which is between $\left[y_{C_{k+2}^{+}}, y_{C_{k+1}^{+}}\right)$, then trajectory $\widetilde{\Pi}(B, t)$ continues to move between trajectories $C_{k+1}^{+} C_{k+2}$ and $C_{k+2}^{+} C_{k+3}$. This process can be continued unlimitedly. Since $\lim _{k \rightarrow \infty} y_{C_{k}^{+}}=y_{P^{+}}$, the intersection sequence of trajectory $\widetilde{\Pi}(B, t)$ and set $N$ will ultimately tend to point $P^{+}$. Similarly, if $B \in\left[y_{D_{k}^{+}}, y_{D_{k+1}^{+}}\right)$, we can also get that the intersection sequence of trajectory $\widetilde{\Pi}(B, t)$ and set $N$ will ultimately tend to point $P^{+}$. Thus the trajectory initiating any point below $A$ ultimately tends to be the unique order one periodic solution $\widehat{P^{+} \widehat{P P}^{+}}$.

The trajectory with any initiating point above $A$ of $N$ will intersect with set $N$ at some point below $A$ with time increasing, so like the analysis above we obtain the trajectory initiating any point above $A$ will ultimately tend to be the unique order one periodic solution $\widehat{P^{+} P P^{+}}$. 


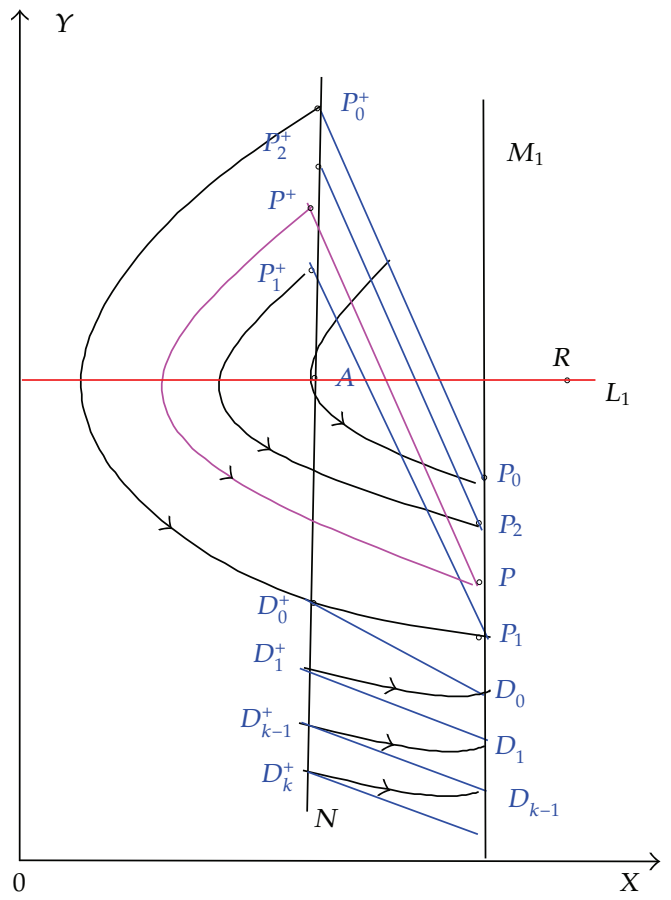

Figure 9

From the above analysis, we know the trajectory initiating any point of $N$ will ultimately tend to be order one periodic solution $\widehat{P^{+} P P^{+}}$. Therefore, the unique order one periodic solution is attractive in the region $\Omega_{1}$. This completes the proof.

Remark 4.2. If system (1.4) satisfies the condition of Theorem 3.1 and that $y_{D_{1}^{+}}<y_{A}<y_{P_{0}^{+}}$, then order one periodic solution with initial point between $A$ and $P_{0}^{+}$is unattractive.

Theorem 4.3. If system (1.4) satisfies the condition of Theorem 3.1 and $y_{P_{0}^{+}}>y_{P_{1}^{+}}>y_{A}$, there exists a unique order one periodic solution of system (1.4) which is attractive in the region $\Omega_{1}=\{(x, y) / x \geq$ $0, y \geq 0, x(0) \leq h\}$.

Proof. Through the derivation of Theorem 3.1, we know there exists an order one periodic solution of system (1.4), whose initial point is between $A$ and $P_{0}^{+}$in set $N$. We assume trajectory $\widehat{P^{+} P}$ and segment $\overline{P P^{+}}$formulate an order one periodic solution of system (1.4), and $P^{+} \in N$ is its initial point satisfying $f\left(P^{+}\right)=0$ (Figure 9). First we will prove the uniqueness of the order one periodic solution.

We take any two points $C_{1}\left(h, y_{C_{1}}\right) \in N, C_{2}\left(h, y_{C_{2}}\right) \in N$ satisfying $y_{C_{2}}>y_{C_{1}}>y_{A}$, then we obtain two trajectories, whose initiate points are $C_{1}$ and $C_{2}$ intersecting with the set $M_{1}$ and two points $D_{1}\left(h, y_{D_{1}}\right)$ and $D_{2}\left(h, y_{D_{2}}\right)$, respectively (Figure 10$)$. In view of the vector field of system (1.4) and the disjointness of any two trajectories, we know $y_{D_{1}}>y_{D_{2}}$. Suppose the points $D_{1}$ and $D_{2}$ are subject to impulsive effect to points $D_{1}^{+}\left(h, y_{D_{1}^{+}}\right)$and $D_{2}^{+}\left(h, y_{D_{2}^{+}}\right)$ respectively, then we have $y_{D_{1}^{+}}>y_{D_{2}^{+}}$and $f\left(C_{1}\right)=y_{D_{1}^{+}}-y_{C_{1}}, f\left(C_{2}\right)=y_{D_{2}^{+}}-y_{C_{2}}$, so we get $f\left(C_{1}\right)-f\left(C_{2}\right)<0$; thus we obtain that the successor function $f(x)$ decreases monotonously on 


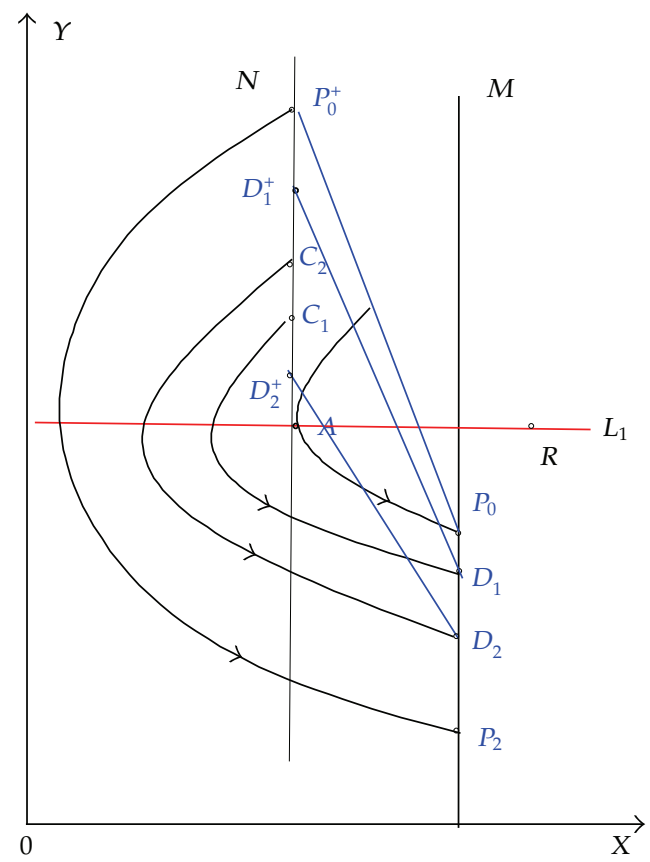

Figure 10

interval $\left(y_{A}, y_{P_{0}^{+}}\right)$of $N$, so there is a unique point $P^{+} \in N$ satisfying $f\left(P^{+}\right)=0$. The trajectory $\widehat{P^{+} P P^{+}}$is a unique order one periodic solution of system (1.4).

Next we prove the attractiveness of the order one periodic solution $\widehat{P^{+} \widehat{P P}^{+}}$in the region $\Omega_{1}$.

Denote the first intersection point of the trajectory with initiating point $P_{0}^{+}$and the impulsive set $M_{1}$ by $P_{1}\left(h, y_{P_{1}}\right)$ and that the corresponding consecutive points are $P_{2}\left(h, y_{P_{2}}\right)$, $P_{3}\left(h, y_{P_{3}}\right), P_{4}\left(h, y_{P_{4}}\right)$, and so forth, respectively. Consequently, under the effect of impulsive function $I$, the corresponding points after pulse are $P_{1}^{+}\left(h, y_{P_{1}^{+}}\right), P_{2}^{+}\left(h, y_{P_{2}^{+}}\right), P_{3}^{+}\left(h, y_{P_{3}^{+}}\right)$, and so forth. In view of $y_{P_{0}^{+}}>y_{P_{1}^{+}}>y_{A}$ and disjointness of any two trajectories, we have that

$$
y_{P_{1}^{+}}<y_{P_{3}^{+}}<\cdots<y_{P_{2 k-1}^{+}}<y_{P_{2 k+1}^{+}}<\cdots<y_{P_{2 k}^{+}}<y_{P_{2 k-2}^{+}}<\cdots<y_{P_{2}^{+}}<y_{P_{0}^{+}}
$$

so $f\left(P_{2 k-1}^{+}\right)=y_{P_{2 k}^{+}}-y_{P_{2 k-1}^{+}}>0$ and $f\left(P_{2 k}^{+}\right)=y_{P_{2 k+1}^{+}}-y_{P_{2 k}^{+}}<0$ hold. As is proved in Theorem 4.1, we can prove $\lim _{k \rightarrow \infty} y_{P_{2 k-1}^{+}}=\lim _{k \rightarrow \infty} y_{P_{2 k}^{+}}=y_{P^{+}}$.

Since the trajectory initiating any point of $\Omega_{1}$ will certainly intersect with set $N$, next we only need to prove the trajectory initiating any point of $N$ will ultimately tend to be order one periodic solution $\widehat{P^{+} P P^{+}}$.

The trajectory with initiating point between $D_{0}^{+}$and $P_{0}^{+}$will intersect impulsive set $N$ with time increasing; under the impulsive effects it arrives at a point of $N$ which is between $\left[y_{P_{2 k-1}^{+}}, y_{P_{2 k+1}^{+}}\right)$or $\left[y_{P_{2 k}^{+}}, y_{P_{2 k-2}^{+}}\right)$. Then like the analysis of Theorem 4.1, we know the trajectory initiating any point between $D_{0}^{+}$and $P_{0}^{+}$will ultimately tend to be order one periodic solution $\widehat{P^{+} P P^{+}}$. 
Assume a point $D_{0}$ of the set $M_{1}$ jumps to $D_{0}^{+}$under the impulsive effect. Set $R\left(D_{0}\right)=$ $D_{1}^{+} \in N$. Assume a point $D_{1}$ of the set $M_{1}$ jumps to $D_{1}^{+}$under the impulsive effect. Set $R\left(D_{1}\right)=$ $D_{2}^{+} \in N$. This process is continuing until there exists a $D_{K_{0}}^{+} \in N\left(K_{0} \in Z^{+}\right)$satisfying $y_{D_{k_{0}}^{+}}<q$. So we obtain a sequence $\left\{D_{k}\right\}_{k=0,1,2, \ldots, K_{0}}$ of the set $M_{1}$ and a sequence $\left\{D_{k}^{+}\right\}_{k=0,1,2, \ldots, K_{0}}$ of the set $N$ satisfying $R\left(D_{k-1}\right)=D_{k}^{+}, y_{D_{k}^{+}}<y_{D_{k-1}^{+}}$. For any point of the set $N$ below $D_{0}^{+}$, it must lie between $D_{k+1}^{+}$and $D_{k}^{+}$where $k=1,2, \ldots, K_{0}$. After $K_{0}+1$ times' impulsive effects, the trajectory from this initiating point will arrive at some point of the set $N$ which must be between $D_{0}^{+}$and $P_{0}^{+}$and then will ultimately tend to order one periodic solution $\widehat{P^{+} P P^{+}}$. There is no order one periodic solution with the initial point below $D_{0}^{+}$.

The trajectory with any initiating point above $P_{0}^{+}$of $N$ will intersect with the set $N$ at some point below $U_{0}^{+}$with time increasing, so the trajectory with any initiating point above $P_{0}^{+}$ will ultimately tend to be order one periodic solution $\widehat{P^{+} P P^{+}}$. There is no order one periodic solution with the initial point above $P_{0}^{+}$.

From the above analysis, we know there exists a unique order one periodic solution in system (1.4) and the trajectory from any initiating point of $N$ will ultimately tend to be order one periodic solution $\widehat{P^{+} P P^{+}}$. Therefore, order one periodic solution $\widehat{P^{+} P P^{+}}$is attractive in the region $\Omega_{1}$. This completes the proof.

\section{Conclusion and Numerical Simulations}

In this paper, a Holling I predator-prey model with state-dependent impulsive control model concerning different control methods at different thresholds is proposed to find a new method to study existence and attractive of order one periodic solution of such system. We define semicontinuous dynamical system and successor function and demonstrate the sufficient condition that system (1.4) has order one periodic solution with differential geometry theory and successor function; by means of sequence convergence rules and qualitative analysis, we prove the attractiveness of the order one periodic solution. These results show that the state-dependent impulsive effects contribute significantly to the richness of the dynamics of the model. The methods of the theorems are proved to be new in this paper and these methods are more efficient and easier to operate than the existing research ones that have been applied to the models with impulsive state feedback control [12-15], so they deserve further promotion.

In order to testify the validity of our results, we consider the following example:

$$
\begin{aligned}
& \frac{d x}{d t}=0.8 x-0.6 x y, \quad x \leq x_{0}, \quad x<h, \\
& \frac{d y}{d t}=-0.2 y+0.3 x y, \quad x \leq x_{0}, \quad x<h, \\
& \frac{d x}{d t}=0.8 x-0.6 x_{0} y, \quad x>x_{0}, \quad x<h, \\
& \frac{d y}{d t}=-0.2 y+0.3 x_{0} y, \quad x>x_{0}, \quad x<h, \\
& \Delta x=-\alpha x, \quad x=h, \\
& \Delta y=-\beta y+q, \quad x=h,
\end{aligned}
$$




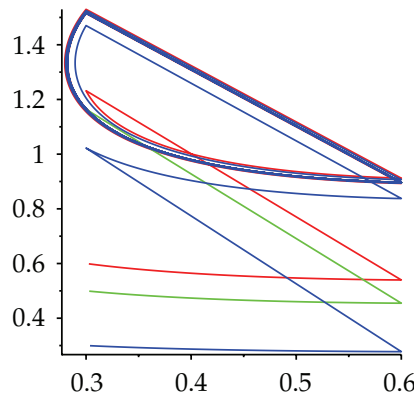

(a)

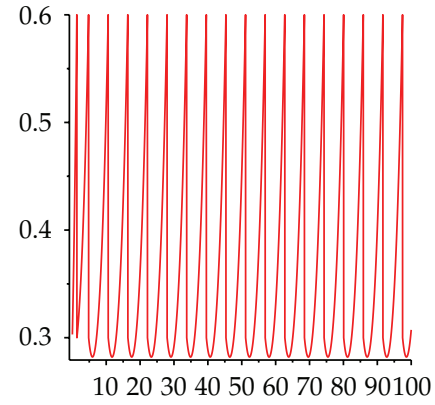

(b)

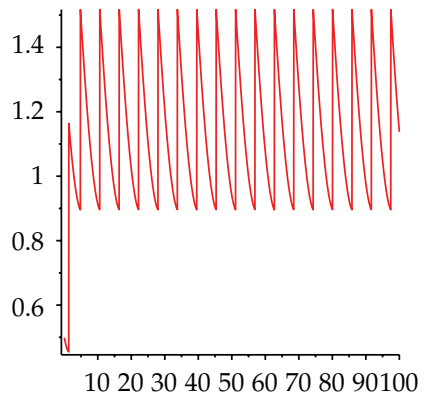

(c)

Figure 11: The time series and phase diagram for system (1.4) starting from initial value $(0.3,0.1)$ (blue), $(0.3,0.5)$ (green), $(0.3,0.6)$ (red), $\alpha=0.5, \beta=0.2, q=0.8, h=0.6, x_{0}=1, h<x^{*}<x_{0}$.

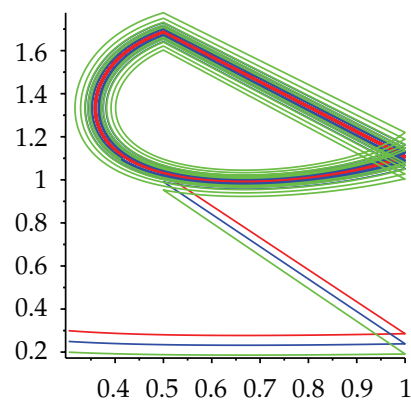

(a)

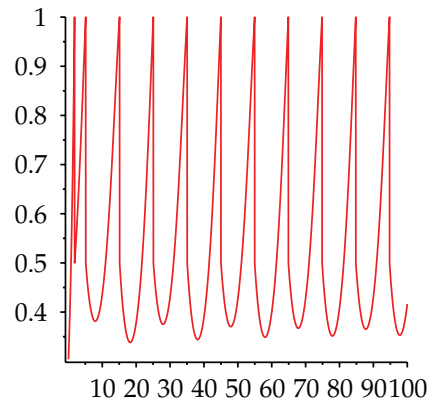

(b)

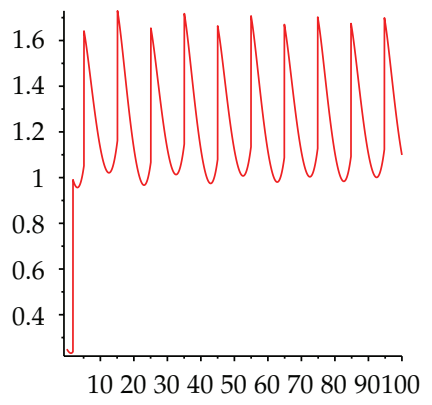

(c)

Figure 12: The time series and phase diagram for system (1.4) starting from initial value $(0.3,0.2)$ (blue), $(0.3,0.1)$ (green), $(0.3,0.15)$ (red), $\alpha=0.5, \beta=0.2, q=0.8, h=1, x_{0}=1.5, x^{*}<h<x_{0}$.

where $\alpha, \beta \in(0,1), q>0, h>0$. Now, we consider the impulsive effects on the dynamics of system (5.1).

Example 5.1. Existence and attractiveness of order one periodic solution.

We set $h=0.6, \alpha=0.5, \beta=0.2, q=0.8, h<x^{*}<x_{0}$, initiating points are $(0.3,0.6)$ (red), $(0.3,0.5)$ (green), and $(0.3,0.1)$ (blue), respectively. Figure 11 shows that system $(5.1)$ has order one periodic solution when the conditions of Theorems 3.1 and 4.1 hold, and the trajectory from different initiating must ultimately tend to be the order one periodic solution. Therefore order one periodic solution is attractive.

Example 5.2. Existence and attractiveness of positive periodic solutions.

We set $h_{1}=1, \alpha=0.5, \beta=0.2, q=0.8, x_{0}=1.5, x^{*}<h<x_{0}$, initiating points are $(0.3,0.3)$ (red), $(0.3,0.2)$ (green), and $(0.3,0.2 .5)$ (blue), respectively. Figure 12 shows that system (5.1) has order one periodic solution when the conditions of Theorems 3.2 and 4.1 hold, and the trajectory from different initiating must ultimately tend to be the order one periodic solution. Therefore order one periodic solution is attractive. 


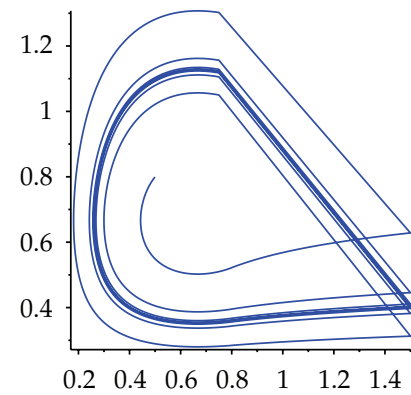

(a)

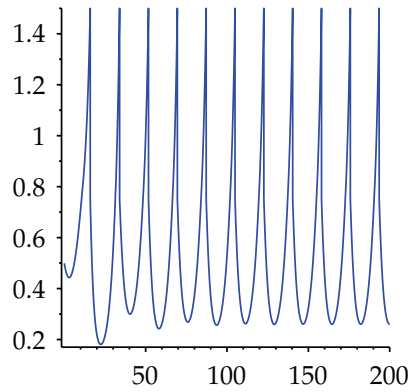

(b)

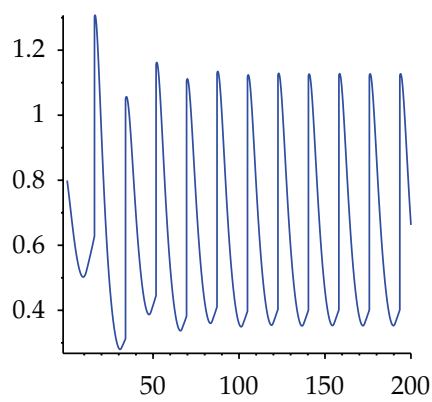

(c)

Figure 13: The time series and phase diagram for system (1.4) starting from initial value $(0.4,0.5), \alpha=$ $0.5, \beta=0.2, q=0.8, h=1.5, x_{0}=0.8, x^{*}<x_{0}<h$.

Example 5.3. Existence and attractiveness of positive periodic solutions.

We set $h=1.5, \alpha=0.5, \beta=0.2, q=0.8, x_{0}=0.8, x^{*}<x_{0}<h$; initiating points is $(0.4,0.5)$. Figure 13 shows that results of Case 3 are valid.

\section{Acknowledgment}

This project was supported by the National Natural Science Foundation of China (No. 10872118).

\section{References}

[1] J. Jiao and L. Chen, "A pest management SI model with periodic biological and chemical control concern," Applied Mathematics and Computation, vol. 183, no. 2, pp. 1018-1026, 2006.

[2] X. Meng, L. Chen, and H. Cheng, "Two profitless delays for the SEIRS epidemic disease model with nonlinear incidence and pulse vaccination," Applied Mathematics and Computation, vol. 186, no. 1, pp. 516-529, 2007.

[3] P. Georgescu, H. Zhang, and L. Chen, "Bifurcation of nontrivial periodic solutions for an impulsively controlled pest management model," Applied Mathematics and Computation, vol. 202, no. 2, pp. 675687, 2008.

[4] X. Meng, L. Chen, and Q. Li, “The dynamics of a impulsive delay predator-prey model with variable coefficients," Applied Mathematics and Computation, vol. 198, no. 1, pp. 61-374, 2008.

[5] B. Liu, Y. Zhang, and L. Chen, "Dynamic complexities of a Holling I predator-prey model concerning periodic biological and chemical control," Chaos, Solitons and Fractals, vol. 22, no. 1, pp. 123-134, 2004.

[6] G. Jiang, Q. Lu, and L. Peng, "Impulsive ecological control of a stage-structured pest management system," Mathematical Biosciences and Engineering, vol. 2, no. 2, pp. 329-344, 2005.

[7] A. d'Onofrio, "Pulse vaccination strategy in the SIR epidemic model: global asymptotic stable eradication in presence of vaccine failures," Mathematical and Computer Modelling, vol. 36, no. 4-5, pp. 473-489, 2002.

[8] J. Jiao, X. Meng, and L. Chen, "A stage-structured Holling mass defence predator-prey model with impulsive perturbations on predators," Applied Mathematics and Computation, vol. 189, no. 2, pp. 1448 1458, 2007.

[9] L. Nie, J. Peng, Z. Teng, and L. Hu, "Existence and stability of periodic solution of a Lotka-Volterra predator-prey model with state dependent impulsive effects," Journal of Computational and Applied Mathematics, vol. 224, no. 2, pp. 544-555, 2009.

[10] J. Jiao and L. Chen, "Global attractivity of a stage-structure variable coefficients predator-prey system with time delay and impulsive perturbations on predators," International Journal of Biomathematics, vol. 1, no. 2, pp. 197-208, 2008. 
[11] X. Meng and Z. Li, "The dynamics of plant disease models with continuous and impulsive cultural control strategies," Journal of Theoretical Biology, vol. 266, no. 1, pp. 29-40, 2010.

[12] C. S. Holling, "The functional response of $\mathrm{p}$ redator to prey density and its role in mimicry and population regulation," Memoirs of the Entomological Society of Canada, no. 45, pp. 1-60, 1965.

[13] Y. Pei, L. Chen, Q. Zhang, and C. Li, "Extinction and permanence of one-prey multi-predators of Holling type II function response system with impulsive biological control," Journal of Theoretical Biology, vol. 235, no. 4, pp. 495-503, 2005.

[14] Z. Xiong, Y. Xue, and S. Li, "A food chain system with Holling IV functional responses and impulsive effect," International Journal of Biomathematics, vol. 1, no. 3, pp. 361-375, 2008.

[15] G. Jiang, Q. Lu, and L. Qian, "Complex dynamics of a Holling type II prey-predator system with state feedback control," Chaos, Solitons E Fractals, vol. 31, no. 2, pp. 448-461, 2007. 


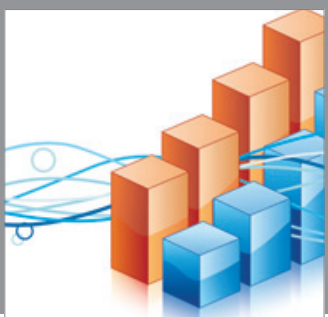

Advances in

Operations Research

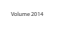

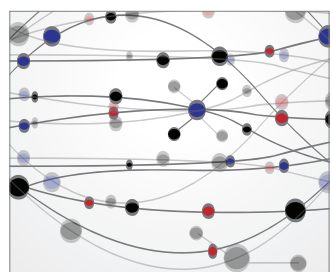

\section{The Scientific} World Journal
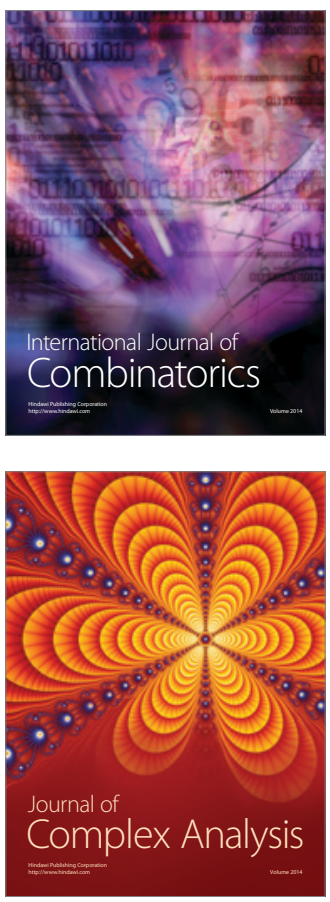

International Journal of

Mathematics and

Mathematical

Sciences
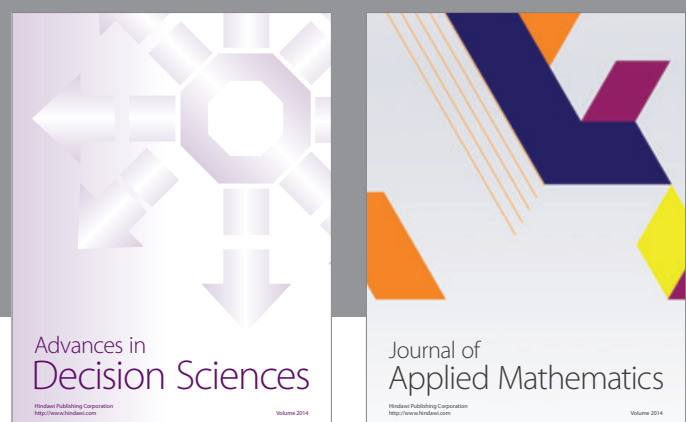

Journal of

Applied Mathematics
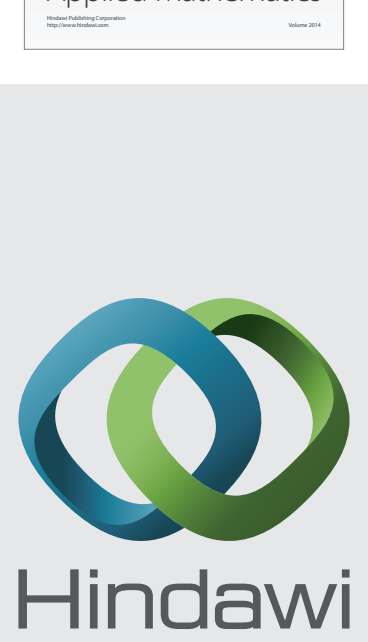

Submit your manuscripts at http://www.hindawi.com
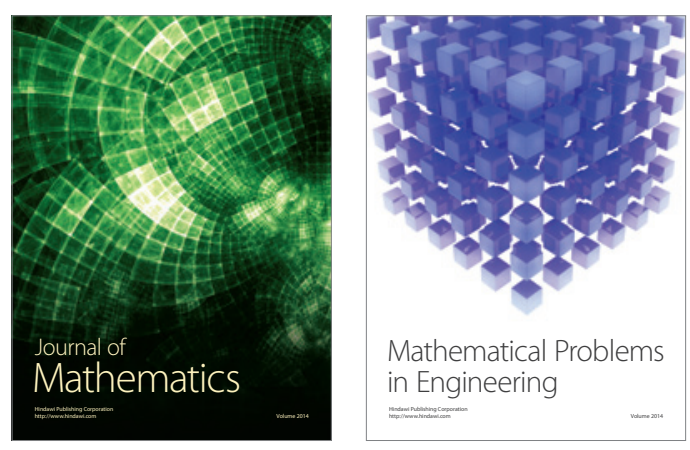

Mathematical Problems in Engineering
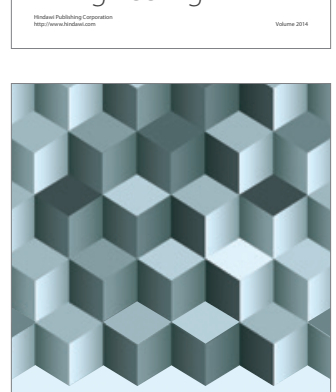

Journal of

Function Spaces
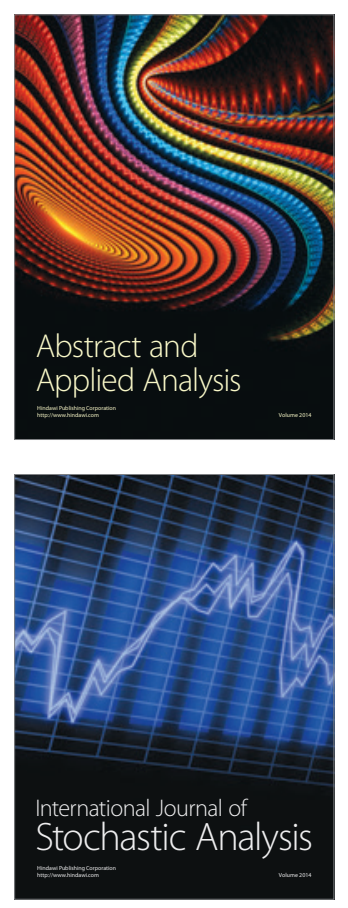

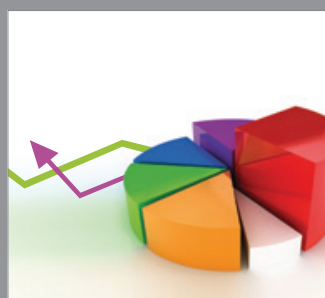

ournal of

Probability and Statistics

Promensencen
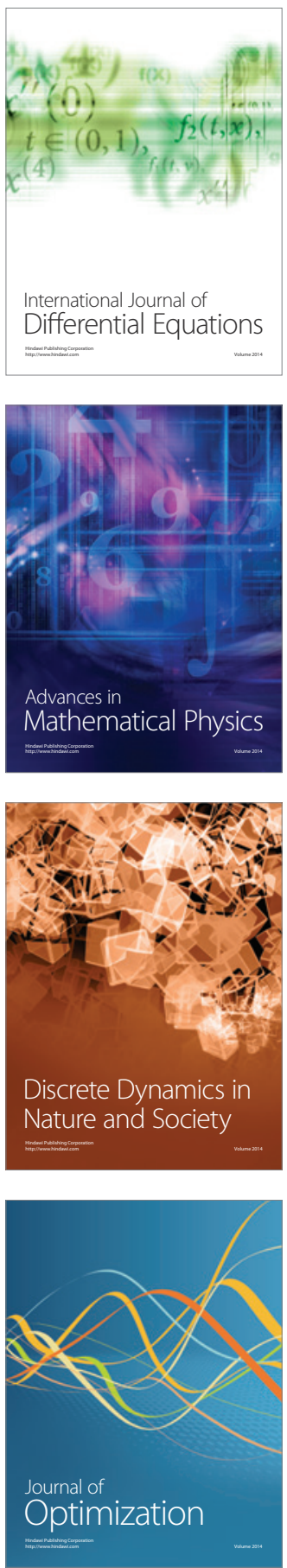